\title{
An extensive study on the quantification of human errors that hampers the performance and construction of structure particularly in geotechnical ambit
}

\author{
Danish Kazmi ${ }^{1}$, Sadaf Qasim ${ }^{2}$, Indra Harahap ${ }^{3}$ and Syed Baharom Azhar ${ }^{4}$ \\ ${ }^{1,2}$ Civil Engineering Department, NED University of Engineering and Technology, Karachi-Pakistan \\ ${ }^{3,4}$ Department of Civil and Environmental Engineering, Universiti Teknologi Petronas, Tronoh-Malaysia
}

\begin{abstract}
Quantification of human errors is generally a baffling work to undertake in any of the industry. With reference to the construction industry, due to scarcity of databases, no widely standard operating procedures until now have been comprehensively adopted. In fact, without methodical database management systems it seems to be quite difficult to quantify the human errors as behavioral aspects of humans are more or less the same in every industry. The main objective of this study is to identify the negligence in construction industry particularly associated with geotechnical engineering in relation to human errors or uncertainties. The concept of human reliability analysis has neither been formally accepted nor thoroughly considered till now despite the consensus that human uncertainty exists. Human reliability analysis proposes different models and methods to pinpoint and quantify human performances as human operations sometime pose big threats to structural reliability. Slope failure is also among the repercussions of flawed design, lapses in construction and poor maintenance of the structures. Among different techniques discussed in this study, this study propounds the model of HEART for human reliability assessment. The reason of selecting HEART model is quite lucid because this model has also been previously tailored by air traffic management and railways. In other words it works well and it has the flexibility to adjust with any other industry.
\end{abstract}

Keywords: Human Errors, Human Reliability Analysis, Structural Reliability Analysis, Probability of Failure

\section{Introduction}

The standard operating procedures to minimize human errors are yet to be established properly and thorough research considering different variables including onsite conditions needs to be undertaken. With reference to the construction industry, due to inadequate databases and information, no proper protocols until now have been adopted.

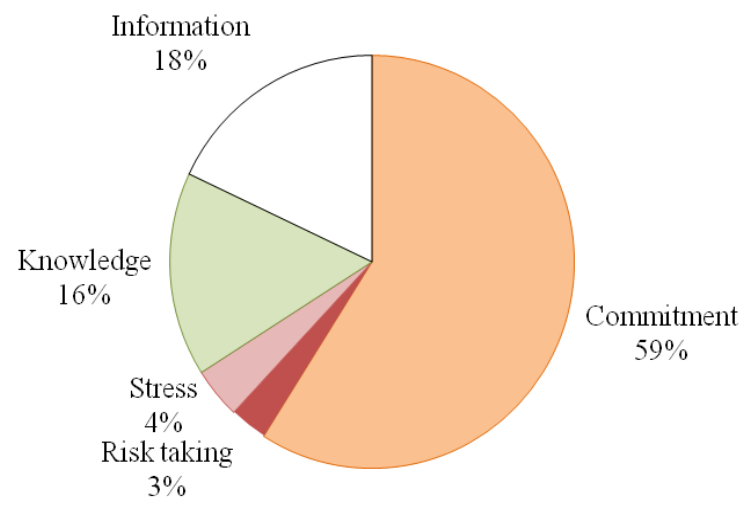

Figure 1:Causes of Faults in Structural Systems(Josephson and Hammarlund 1996a)

Hence, without proper database systems it is quite elusive to quantify the reasons for human errors and suggest remedies for their curtailment. In geotechnical engineering, slope failure is also among the consequences of deficit design, flaws in construction or poor maintenance of the structures used to strengthen the slopes(Jamaluddin 2006). Malaysian report itself admitted that, among the 49 major cases of landslides 88 percent are accredited to manmade slopes (JKR 2009). (Gue and Tan 2007) also approved that along with poor designing, incompetency, negligence, raw input data are the responsible agents of these slope failures. It is a fact that human uncertainties are yet to be completely recognized and valued. This study aims to analyze these uncertainties in the construction sector especially in the geotechnical field. Therefore, due to various governing 
An extensive study on the quantification of human errors that hampers the performance and ..

factors, the human reliability analysis or likelihood of human errors in different phases of design, construction and maintenance needs be performed.

Consideration of human errors whether it is technical based or attitude dependent has already been predicted or quantified by different estimation techniques like Human error assessment rate technique (HEART), Technique for human error rate prediction (THERP) and Success likelihood index method (SLIM) in nuclear power industry and chemical industry. Already in hand data bases of nuclear power industry and chemical industry in one way helping other industries to check the influence of human errors. It is obvious now that role of humans is pivotal not only in safe operation of complex industrial plants but also in airways, railways and in construction industry.

The objectives of this study are:

a. To accentuate the role of human errors by discussing human error models and human reliability analysis techniques.

b. To investigate the potency of human reliability analysis approaches in determining the behaviour of human performance.

c. To confirm the need of human reliability analysis in construction and geotechnical industry by analyzing numerous catastrophic failures into discussion

\section{Human Error Models}

Different types of human error models are discussed in detail below as human errors are divided into two sections, slips and mistakes. Slips are referred as unintentional errors, defined as unintended performance of an action. Mistakes lie under the category of deliberate actions means; knowingly do inappropriate selection or choice in the system.

\section{Rasmussen Srk Model}

The model (Figure 2) has taken human errors into three segments. Skill based, rule based and knowledge based (Rasmussen 1982)

a. Skill based: acts involve the most minuscule level of cognitive job for example doing much known operations.

b. Rule based: it needs more concentration as compared to skill based acts. An example of the skill required at this point is to remind or recall the suitable rules for non familiar jobs.

c. Knowledge based: acts under this level require full concentration and consciousness. Mental capabilities / full knowledge have to be consumed to analyse or solve the issues.
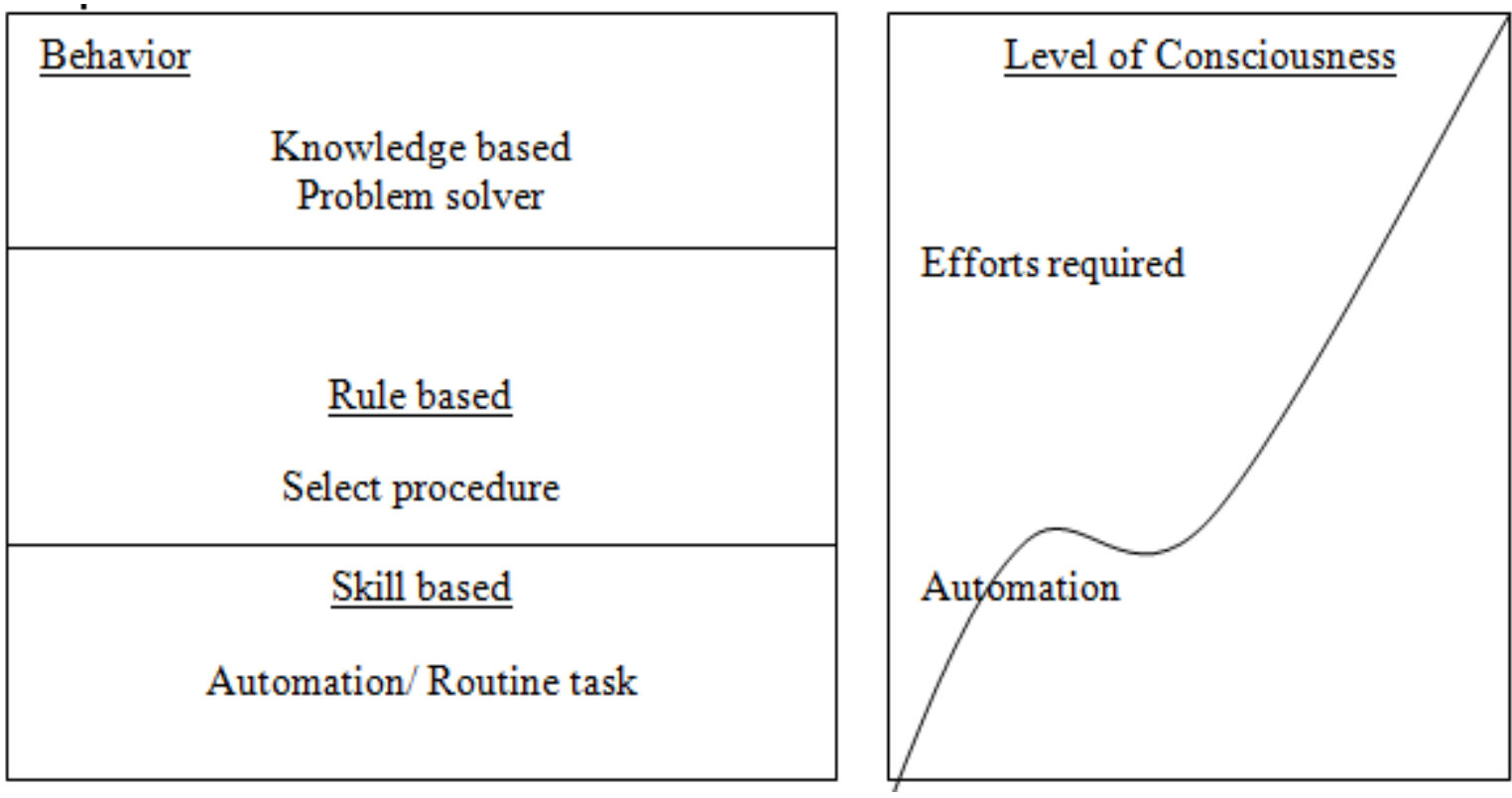

Figure 2: SKR Model (Rasmussen 1982)

\section{Reason Model (1990)}

In actual violations are deliberate actions, knowingly deviated from safe guidelines or procedures. Unintentional acts may be disordering, mistiming, intrusion reveal or omission. It all lies under slips. Lapse is basically forgetting, omitting planned issues or place-losing. Intended acts shows misapplication of rules, 
standards or avoiding specifications. Violations are on routine or on an exceptional basis. The summarized view of violations/unsafe acts is shown in Figure 3.

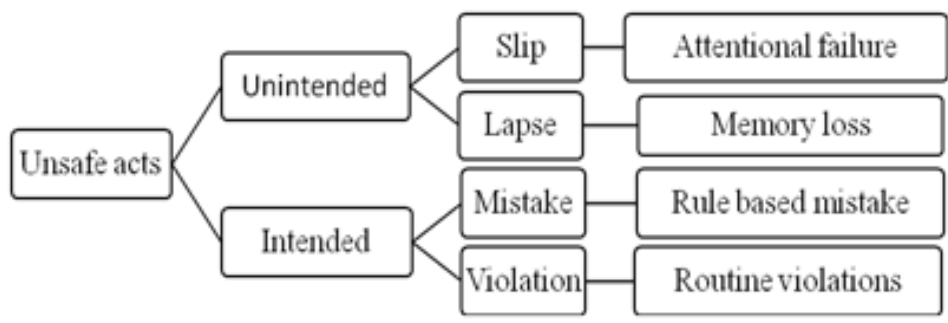

Figure 3: Unsafe Acts (Reason 1990)

\section{Human Reliability Approaches}

The approaches to calculate human reliability have occupied two categories: one looped with databases and other totally relying on expert's opinion. The first category consists of those techniques which has already in hand generic error probabilities. These generic probabilities are than manipulate by the evaluator to extrapolate from the generic data to the particular scenario being considered. Manipulation is usually stood on assessor's judgment of situation governing Performance Shaping Factors or Error Producing Conditions. Techniques lie in second category are not so structured, totally relying on personal communication and asking to estimate the probabilities of the specific situation. Examples of these category techniques are Absolute Probability Judgment (APJ) and Paired Comparison (PC). Success Likelihood Index Method also belongs to second category but this technique follows a structured pattern. The generation of HEPs may therefore arise through expert's opinion or by combination of assessor's manipulation and interrogation of quasi-databases (Kirwan 1998).The most commonly used techniques are described below.

\section{Technique For Human Error Rate Prediction (Therp)}

This technique uses a database of error probabilities tailored by the assessor by using Performance Shaping Factors. The process of quantification is shown in Figure 4. The key points of this technique are:

a. Decomposition of tasks into elements and allot nominal HEPs

b. Find out the influence of PSFs on each element

c. Determination of effects of dependence between tasks

d. Using Event tree analysis for modelling and quantifies total task by HEP

The selection of nominal HEPs according to the considered task/element is carried out with reference to Chapter 20 of the handbook of THERP. It is one of the followed techniques used in Nuclear Power Plant and Reprocessing industries (NP\&R) in U.K adapted from (Kirwan 1996).

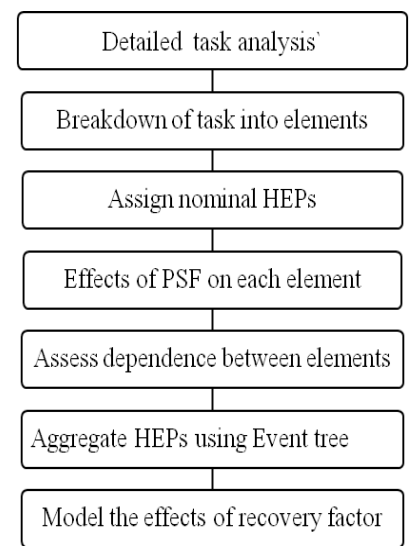

Figure 4: THERP Process (Kirwan 1996)

\section{Human Error Assessment Rate Technique}

There are more than 30 Error Producing Conditions (EPCs) provided in HEART technique for matching with identified PSFs related with the focused task (Performance Shaping Factors). Most of them are very common in use namely time stress, unfamiliarity, poor feedback, poor procedures etc. Calculation of HEART is dependent on generic error probability and related EPCs. Generic error probability has to be selected from the given criteria A-H according to focus situation EPCs carrying a maximum affect value, which has to be 
An extensive study on the quantification of human errors that hampers the performance and ..

changed with the estimated proportion. Proportion of this value has been estimated by expert's opinions and a mean value is applied (Fig 5).

As compared to other error rate prediction techniques it is quite easy. It requires only the perception of the user; no detailed calculations are involved in it. Its validity and accuracy is already confirmed through a large scale study of 30 tasks (Kirwan 1998). This can be applied to any industry where the human reliability has to be checked. No doubt due to insufficient data results may not be so sophisticated but at least in the scenario where human factors are more influential can be assessed by applying HEART technique. This technique serves in determining human error probabilities(Kirwan 1998).

Human error probability (HEP) is defined as number of errors occurred divided by the total number of opportunities to occur. Nominal HEPs can be derived out by using a record of total events with number of events occurred due to human errors (without considering human factors). This can be called as raw human error probability. In calculating basic human error probabilities (HEPs) proportion of Error Producing Conditions (EPCs) must be included.

A simple process is followed on the basis of the following formula(Kirwan 1998)

$\mathbf{H E P}=\mathbf{G T T}[(\mathbf{E P C}-\mathbf{1}) \times \mathbf{A P O A}+\mathbf{1}]$
EPC $=$ Error Producing Condition
GTT $=$ Generic Task Type
APOA $=$ Assessed Proportion of Maximum Affect

$\mathrm{APOA}=$ Assessed Proportion of Maximum Affect

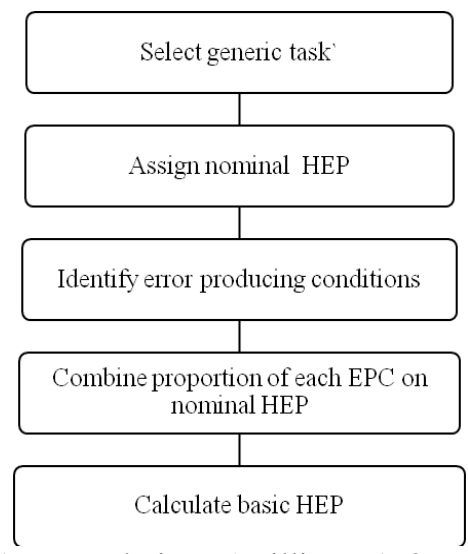

Figure 5: HEART Technique (Williams 1986; Williams 1988)

\section{Controller Action Reliability Assessment (Cara)}

This technique is basically on the format of HEART technique(Williams 1986). It is utilized to quantify human performance in Air Traffic Management. According to focused environment CARA (Gibson and Kirwan 2006; Redmill, Anderson et al. 2007) modified HEART technique by generating its own GTTs and EPCs. HEART has been selected as a model because it has been the subject of confirmation exercises (Kirwan, Kennedy et al. 1997) and the significance and compliance to different domains is propping up by recent developments of HEART in nuclear industry (Edmunds, Huw Gibson et al. 2008) and railways (J.W. Kim, W. Jung et al. 2006).

\section{IX. $\quad$ Success Likelihood Index Method (Slim)}

It is a pure judgment base but structured technique, without experts' opinions and discussions it can't be executed. Visualize the event and sub events and the rating and the weighting of the concerned PSFs all requires expert's panel. Consistency level between their decisions will also not to be overlooked. It is very easy to define but not easy in execution. On the basis of PSFs ratings and weights Success Likelihood Index (SLI) is determined. SLI in original is the product of weight and rate of single event. Conversion of SLI values into probabilities also needs logarithmic relationship. This technique has now computer version named MAUD. MAUD stands for multi Attribute Utility Decomposition helps to cover the biases of judges' opinions and decisions (Bell and Holroyd ; Grozdanovic 2005).

Human Error Probabilities Index (HEPI) is also predicted through this technique of SLIM (Figure 6) in offshore events. Due to scarcity of database, a panel of 24 experts is selected to work out the rating and weighting of the governing PSFs. PSFs are stress, complexity, training, experience, event factors and atmospheric factors, Rating scale or weighing criteria for PSFs is provided as a guide to direct the experts (DiMattia, Khan et al. 2005) .

For a specific muster action, weight of each PSF is modified by dividing the sum of the weights of all the PSFs for that action. The final output success likelihood index of the specific action is then obtained by

DOI: $10.9790 / 1684-1304053343 \quad$ www.iosrjournals.org $36 \mid$ Page


multiplying the rating and modified weight of that particular PSF. For specific action six values of SLIs are determined, the summation of all these 6 values gives a total SLI value of that considered action. The higher the SLI value the greater the chances of successfully accomplishing the action. After having the SLI values, next step is to estimate the HEPs by taking logarithmic relationship:

$\log \left(\mathbf{P O S}_{\mathbf{i}}\right)=\mathbf{a}\left(\mathbf{S L I}_{\mathbf{i} \mathbf{m}}\right)+\mathbf{b}$

$\left(\mathrm{POS}_{\mathrm{i}}\right)=1-\mathrm{HEP}_{\mathrm{i}}($ Probability of success for action $\mathrm{i})$

$\mathrm{SLI}_{\mathrm{i}, \mathrm{m}}=$ arithmetic mean of Success likelihood index values of action $\mathrm{i}$

$\mathrm{a}, \mathrm{b}=$ constants

Constants ' $a$ ' and ' $b$ ' needs two basic HEPs of an action carrying highest and lowest SLI values. For

Basic Human Error Probabilities (BHEPs) three approaches are there.

a. Empirical BHEPs from limited available master data

b. Elicited HEPs from randomly selected subset of elicited review team

c. THERP data of(Swain and Guttmann 1983) and Kirwan (1994)

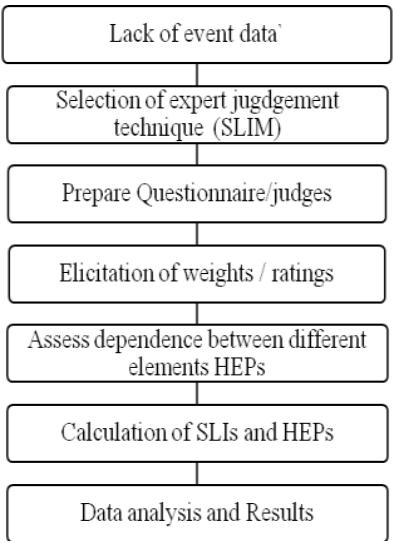

Figure 6: SLIM Technique (Grozdanovic 2005)

Among several techniques, HEART, SLIM and THERP are widely applied in taking nuclear power plants and offshore scenarios. These techniques no doubt differ with each other at different levels (Table 1) like suitability, validity, accuracy and effectiveness (Kirwan 1994).

Table 1:Comparisons of Techniques (Kirwan 1994)

\begin{tabular}{|l|l|l|l|}
\hline Criteria & THERP & HEART & SLIM \\
\hline Accuracy & Medium & Medium & Medium \\
\hline Validity & Medium & Medium/High & Medium/High \\
\hline Usefulness & Medium & High & High \\
\hline Effective use of resources & Low/Medium & High & Medium \\
\hline
\end{tabular}

X. Qualitative Simulation With Human Reliability Analysis

The core intention of HRA is to accurately weigh up risks generated from human error and determine ways to mitigate human error. In special cases, the outcome objective of Human Reliability Analysis should be to work for reasons of reliability decline produced from human error and approaches to overcome them(Kirwan 1994). As a result, it shows divergence from HRA into human error analysis (HEA). A complete HRA process must have the following steps:

a. Recognition and description of human error

b. Quantification of human error probability

c. Analysis of human error modes and effects

d. Design and authentication of protective measures for human error.

Human reliability analysis in complex industrial processes is in front of difficulties, such as deficient knowledge utilization and simplex methodical means etc. The research of (Long, Hongwei et al. 2009) clarifies basic meanings and current progress position of human reliability analysis and qualitative model correspondingly, and then on the foundation of it, considering existing deficiency of human reliability analysis approaches, the research of (Long, Hongwei et al. 2009) in actual publicize the essentiality to relate qualitative simulation with human reliability analysis by proposing framework (Fig. 7). In the proposed framework factors of task $(\mathrm{T})$, man machine interaction $(\mathrm{Q})$, training level $(\mathrm{T})$, environment $(\mathrm{E})$ and work time $(\mathrm{t})$ has been compared in correspondence with actual and expected ability. It supports qualitative simulation of exposes returns in making uncertain message and "Deep" knowledge in the fields of supporting analysis and decision 
making and has grow to be a kind of effectual methods in fixing incomplete knowledge. There is a sense inevitability and genuine achievability to pertain qualitative simulation into HRA.

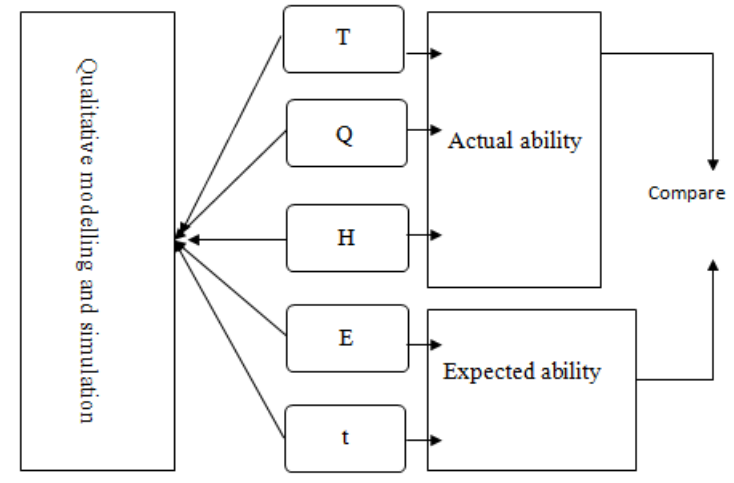

Figure 7: Framework for Qualitative Study (Long, Hongwei et al. 2009)

\section{Human Reliabity Analysis Applications}

A case history of existing nuclear power plant in connection with human reliability assessment is highlighted. The HRA has taken up all steps from problem description and task analysis through error identification and quantification to impact assessment error minimization, quality assurance and documentation. In the study of (Kirwan, Scannali et al. 1996) a methodology of HRA with respect to probabilistic safety assessment (PSA) is proposed. The primary focus of PSA is on operational or equipment weaknes ses/flaws in nuclear power plant and to work out plans of how to wipe out these weaknesses. HRA along with human factor or ergonomics also has the capacity to read design procedures, training vulnerabilities and to indicate ways for its rectification. This is called error reduction. Quantitative evaluation of all the contributors to risk can be assessed by Fault tree or event tree analysis. Error reduction can target only particular errors. A specific Performance shaping factor is considered only, when, it repeatedly originating a number of disparate errors. This type of error reduction approach is referred as strategic error reduction. Cognitive errors examples are wrong diagnosis or late diagnosis. Errors of commission are those actions which are not required by the system. These errors are of prime importance in nuclear power plant industry. As incident of Three Mile Island divert the attention towards this aspect also.In connection with crew and operators action of nuclear power plant industry a cognitively supported human reliability assessment technique for calculating the HEPs has been discussed (Blackman, Gertman et al. 2008). The method Standardized Plant Analysis Risk - Human Reliability Analysis (SPAR-H) is used to quantify human performances at nuclear power plants. SPAR-H is the product of Technique for Human Error Rate Prediction (THERP) and. Accident Sequence Evaluation Program (ASEP). Human Reliability Analysis Procedure (ASEP) is also the simplified version of THERP. SPAR-H method focuses on two aspects, diagnosis or action. Action oriented task includes operations, calibrations testing etc. Diagnosis tasks refer to planning and prioritizing activities, using knowledge and experience to read the existing conditions. This method has pre defined nominal HEPs which has been modified by the application of related PSF multipliers. (Boring and Blackman 2007) presented the history of these multipliers. SPAR-H uses eight PSFs having multipliers typically corresponding to nominal, degraded and severely degraded human performance for individual PSFs. In the absence of PSFs nominal HEPs for diagnosis and action refers to 1E-2 and 1E-3 respectively. Till now HRA proposed 50 PSFs as used in IDAC (Mosleh and Chang 2004; Mosleh and Groth 2009) model, SPAR-H methods according to nuclear power plant requirement selected initially 6 than 8 PSFs. Selected PSFs includes:

a. available time

b. stress and stressors

c. experience and training

d. procedures

e. Complexity

f. fitness for duty

g. work processes

h. human machine interaction

Few of the PSFs and its multipliers are given below as an example. Referring to experience and training; three levels low, nominal and high are in use to set the multiplier. High level shows extensive experience and perfect knowledge to tackle the situations and a value of 0.1 is used to modify HEPs. In case of low and nominal levels multipliers of 10 and 1 are taken. In this governing PSF of experience and training period of 6 months or more than 6 months are adjusted with low and nominal levels. Stress and stressors also have three main levels; extreme, high and nominal carrying values of 5, 2 and 1 respectively. Environmental 
An extensive study on the quantification of human errors that hampers the performance and ..

factors such as heat, noise poor ventilation are also the source of stress to operators. Talking about fitness for duty again three levels unfit, degraded fitness and nominal exists with this PSF. First level of unfit shows no margin as individual is unable to perform, the probability of failure in this case is $100 \%$. For rest of the two levels, multipliers of 5 and are fixed. Work processes also carry three levels of poor, nominal and good with multipliers of 2,1 and 0.8 respectively.

\section{Need Of Human Reliability Analysis In Construction And Particularly In Geotechnical Industry}

According to its mode of occurrence human errors in structural reliability are classified as:

- Conceptual errors

- Errors in execution

- Intended errors

Conceptual errors are basically unintended errors, due to insufficient knowledge. It is defined as the departure from the accepted practices unknowingly. Execution errors are the result of unpremeditated departure from the conceptual model existing in the mind of the contractor or designer. Intended errors are planned errors (El-Shahhat 1995). In discussion of 500 failure cases 58\% attributed to design errors $38 \%$ is originated from construction, $4 \%$ in operation. Approximately half of the errors are from design and second half refers to construction. Three causes are identified, absence (12\%), ignorance (33\%) and rejection of modern/ current technology (55\%) (Sowers 1993). (Melchers 1984) reported through evidences that structural resistance suffers due above mentioned errors. Human errors in design and construction process turn out a low resistant structure than actually expected. It is also agreed that a large share of structural failures are due to human error in the design stage of any of the structural project, and many of these failures could have been ward off if there had been passable design checking (Stewart and Melchers 1989). Results are furnished from surveys probing the usefulness of three classical design-checking strategies: self-checking, independent detailed design checking, and overview checking. Following a reconsider of present work in this locale, fitting mathematical models, which scan the effects of error magnitude, times, and experience, are wished-for for each design checking process. (Frangopol 1986) has taken human errors into probabilistic models. At first error free reliability index is computed than it modifies by accounting human errors. In calculating structural risk always start from an ideal case of having error free system. (Lind 1982) has also proposed three mathematical models (like discrete model, error filter and elimination model and error combination) model to counter human errors for maintaining maximum reliability of the structure. In relation with structural failures, one very debatable observation is that their occurrence is knowledge based. Unknown knowables and unknown unknowables are two categories of this knowledge based challenges (Bea 2006). The first category is already discussed by (Sowers 1993) as rejection or technology misuse. Second category belongs to limitations in knowability. Potential of an engineer is limited for the extraction of knowledge. (Sowers 1993; Bea 2006) discussed four quality objectives serviceability (i=1), safety $(\mathrm{i}=2)$, compatibility $(\mathrm{i}=3)$ and durability $(\mathrm{i}=4)$ with respect to six life cycle processes concept development, design, construction, operation, maintenance and demolition. In this aspect failure probability is:

$\boldsymbol{P}\left(\boldsymbol{F}_{i}\right)=\left(\boldsymbol{D}_{i} \geq \boldsymbol{C}_{i}\right)$

$D_{i}=$ the demand placed on the system

$C_{i}=$ capacity of the system to fulfill the demand

The probability of failure with respect to quality attribute $(i)$ due to essential nature $(I)$ or non essential nature factors $(E)$ is:

$\mathbf{P}\left(\mathbf{F}_{\mathrm{i}}\right)=\mathbf{P}\left(\mathbf{F}_{\mathrm{iI}} \cup \mathbf{F}_{\mathrm{iE}}\right)$

Non essential nature extrinsic factors can also be excluded or managed through Quality Assurance and Quality Control QAQC. Base rates of extrinsic factors, category of Performance Shaping Factors are necessary features of Quality Management Assessment System (QMAS). Qualitative grading of QMAS and its conversion into quantitative Performance Shaping Factors (PSFs) is shown in fig 8. 


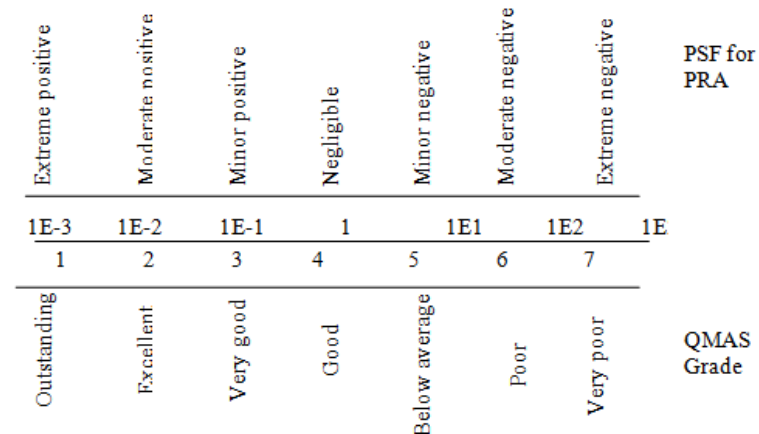

Figure 8: Quality Management Assessment System (Bea 2006)

The European Federation of National Maintenance Societies described maintenance as: All actions which have the goal of reinstating or retaining an item in or to a state in which it can execute its essential function. The actions consist of all technical, administrative managerial and supervision actions. (Janney 1986) defined structural failure as: "The reduction of the capability of a structural system or component to such degrees that it cannot safely to serve its intended purpose".

Human factors which contribute in structural failures/errors are given in Table 2. Three very basic tasks are selected by (Melchers 1984) to search the evidence that whether the involvement of humans exists or not in deficit design for any structure that predominantly governs are given below.

1. Table look up

2. Numerical work

3. Ranking of numbers

These tasks comprise of member selection from tables (Task 1), simple stress calculations (task 2) and matching with pre defined criteria (Task 3). The errors are classified as random or gross errors. Gross errors reflect in those values which depart more than $2.5 \%$ from correct values. On the basis of these tasks error rates are determined which is quite agreeable when compared with the range of values of other error rates of psychomotor tasks.

Table 2: Structural Failures/Errors (Melchers 1984)

\begin{tabular}{|c|c|c|c|}
\hline Concept errors & \multicolumn{3}{|c|}{ Random/systematic } \\
\hline \multirow{8}{*}{$\begin{array}{l}\text { Design } \\
\text { errors }\end{array}$} & Modelling & & Random/systematic \\
\hline & \multirow[t]{2}{*}{ Calculation } & Decimal & \begin{tabular}{l|l} 
Round off & Computational
\end{tabular} \\
\hline & & \multicolumn{2}{|r|}{ Random/systematic } \\
\hline & Discretization & \multicolumn{2}{|r|}{ Random/systematic } \\
\hline & Table - use error & \multicolumn{2}{|r|}{ Random/systematic } \\
\hline & Code interpretation & \multicolumn{2}{|r|}{ Random/systematic } \\
\hline & Code validity & \multicolumn{2}{|r|}{ Random/systematic } \\
\hline & Data interpretation & \multicolumn{2}{|r|}{ Random/systematic } \\
\hline $\begin{array}{l}\text { Design-construction } \\
\text { interface }\end{array}$ & \multicolumn{3}{|c|}{ Random/systematic } \\
\hline Construction errors & \multicolumn{3}{|l|}{ Random/systematic } \\
\hline Usage errors & \multicolumn{3}{|l|}{ Random/systematic } \\
\hline
\end{tabular}

Even the study has been condemned due to its unsophisticated pattern: taking students as subject to fill the questionnaire, very few data points etc but the results are more or less similar. No doubt care has been taken like vague replies are not entertained, time frame is focused and the purpose of this task has not been communicated to the students.By an event tree process having all the design steps, taking into account all the branches implied by omission errors and the variability due to commission errors. The reliability of design along with human error inclusion can be estimated by this method. The total probability of failure is obtained by adding the probabilities determined for descending via all possible combinations in event tree.

In few studies human errors has been discussed in an indirect manner, for example among 143 bridge failures, 70 is claimed due to foundation movement, 22 by unsuitable or defective material and so on (Smith 1977). The researcher does not point out human errors. (Matousek 1977) in contrast to (Smith 1977) concluded that human errors always there in a sample of 800 cases. An example of the catastrophic failure of Kwun Lung Lau landslide in Hong Kong is also the input of human uncertainty(Morgenstern 1995). Most of the cases of Malaysian landslides for example Highland Towers 1993, Bukit Antarabangsa 1999, Bukit Antarabangsa 2008 are also the ending are also the result of improper safety factor, poor or non maintained drainage facilities(JKR 2009).Discussing about the documentation and analyses of construction failures (Yates and Lockley 2002) 
An extensive study on the quantification of human errors that hampers the performance and ..

reported the division of construction failures into two categories: technical and procedural, technical causes/failures are real material proximate causes like improper soil compaction results in excessive settlement. Procedural causes directly involve humans as due to miscommunication or flaws in designing and construction. These procedural errors are in actual responsible for physical failures. Organizational issues are also related here as when soil testing laboratory fails to check the compaction of the soil. The division of structural failures has been taken place into three general categories of functional, safety ancillary and its belongs to causes into five general areas (Thornton 1985):
a. Deficit design
b. Construction flaws
c. Material deficiencies
d. Organizational/administrative shortcomings
e. Improper/poor maintenance

Observational method (Peck 1969) is best suitable for every ongoing construction but it only suits when design alterations are possible. (Haydl and Nikiel 2000) also pinpoint by taking case histories which refers construction failures, due to redundant bracing, wrong assumption of the design engineer, improper sequences or at an eleventh hour changes. Through field survey and unstructured interviews the fact is exposed that managerial influence is more dominating in defects of construction industry (Refer Table 3). Errors committed during operations and supervisions are not only one man show. In a construction industry methodical literature review from technological, social science and management perspective has been directed to develop three stage models of error sources (Atkinson 1999).

Table 3: Construction Industry Error Sources (Atkinson 1999)

\begin{tabular}{|l|l|}
\hline \multirow{4}{*}{ Primary } & Knowledge training/education \\
\cline { 2 - 2 } & Selection of knowledgeable personnel \\
\cline { 2 - 2 } & Self inspection of task \\
\hline \multirow{4}{*}{ Managerial } & Checking work \\
\cline { 2 - 2 } & Dividing responsibilities \\
\cline { 2 - 2 } & Controlling change \\
\cline { 2 - 2 } & Controlling concurrent working \\
\cline { 2 - 2 } & Communication \\
\hline \multirow{4}{*}{ Global } & Organizational culture \\
\cline { 2 - 2 } & Economic pressure \\
\cline { 2 - 2 } & Time pressure \\
\cline { 2 - 2 } & Political pressure \\
\cline { 2 - 2 } & Societal pressure \\
\hline
\end{tabular}

The findings prove that communication is the most significant source of error production. It is rated very high in lieu of other error sources. Primary factors are throughout important but at the management level its importance is highly concerned. One interesting fact also comes to know that experience without knowledge/qualification has got no weight age. Most often inexperienced but qualified personnel take right/ better decisions. Inexperienced managers having sound educational background reported lower level of defects. The safety of reinforced concrete structures is of major concern during construction. Human errors types such as inadequate pouring of concrete, untimely removal of formwork, concrete cover inadequacies, poor quality of supervision create major negative effects. The Swedish study (pinpointed slightly, in Section 1.1) reveals the causes of quality fault costs in building projects(Josephson and Hammarlund 1996a; Josephson and Hammarlund 1996b). Seven different building projects are studied and overall of 2879 faults are detected. Each fault is penned along with its essential information regarding explanation of fault, building component part involved, fault origin, i.e. which part of the building process was responsible, primary cause fault type. The faults are categorized according to type of work performed, with approximately 120 faults beginning from gathering of formwork, 70 faults from reinforcement task and 180 faults from concrete casting.

Examples of faults starting from connecting of formwork:

a. Wrong position of construction joint (cause: knowledge)

b. Recess in construction joint not carried out (cause: commitment)

Examples of faults coming out from reinforcement:

a. Wrong reinforcement approved (cause: commitment)

b. Recess strip mislaid (cause: information)

Examples of faults basis on concrete casting tasks:

a. Wrong quality of concrete transported (cause: commitment, information)

b. Casting flaws (cause: commitment, unavoidable due to existing knowledge, method and equipment) 
c. Improper curing of concrete wall (cause: time pressure)

One of the study presented probabilistic and human reliability models. The models used to estimate the system risk during construction due to human errors. Error control measures in terms of inspection are judged by taking two cases: system risk before inspection and system risk after inspection (Fig 9). Sensitivity analysis is also executed to conclude the effects of individual or multiple errors on system. A common issue in reinforced concrete constructions is that, a same error crop up more than once. System risk has been evaluated on three shoring systems denoted as $2 \mathrm{~S}, 2 \mathrm{~S} 1 \mathrm{Rand} 3 \mathrm{~S}$. It is clearly speaks out from Figure 4.10 that changes in the initial errors after inspection is around 70 to $80 \%$. This shows that some errors can be easily sort out if proper follow up of the work exists. Final error human reliability models are used for Sensitivity analysis. Through sensitivity analysis it is noted that due to inaccurate concrete cover, system risk increases up to $45 \%$ among other error rates. This is the most alarming increment in system unreliability. Due to poor concrete workmanship system becomes unreliable up to $25 \%$ but very minute effects are measured due to premature removal of shoring. This is maybe due to low error magnitude. In case of combination with different sort of errors premature removal of shoring poses dramatic change. The system risk is lowered down up to $10 \%$ if the above mentioned particular error is not taken into consideration. System risk is in actual the probability of structural failure/ collapse during construction due to human errors.

It is also quite clear now that construction cycle will affect the system risk as punching shear is dominant for final error risk system. Concrete strength will become lower due to cut short of construction cycle.

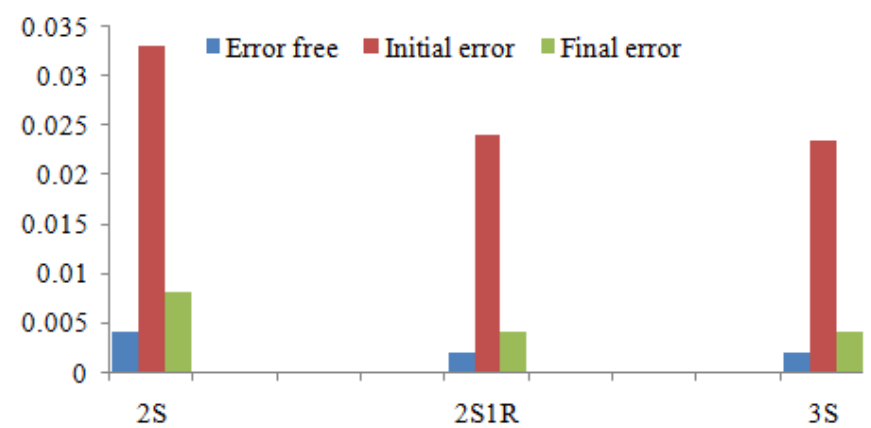

Figure 9: Human Errors Before And After Inspection (Epaarachchi and Stewart 2004)

\section{Conclusion}

It is now evident that neglecting the role of human performances and human errors could lead to potential losses during the construction process. Despite of some existing research which also includes assessment from indirect perspective, there is an immense need to directly address the human performance error and suggest the measures to mitigate it. In addition to that, there is enormous requirement to devise standard operating procedures and protocols to minimize human performance errors that should be feasible for a range of situations.

The discipline of geotechnical engineering with expertise in slope engineering is increasingly gaining prominence nowadays due to increasing number of landslides. Most of the slope failures take place on manmade slopes and this is basically the result of erroneous design, lapses in construction and inadequate maintenance. Other studies have also proved that poor deign, incompetence, negligence during work and raw input data sparks these slope failures. Till date, this fact is not accepted truly that uncertainties related to human factors needs to be properly addressed in order to beef up the overall construction process especially in geotechnical engineering applications. In a broader spectrum, reliability of the slopes needs to be evaluated not only through structural probability of failure but also by the probability of failure due to human errors as structural probability of failure is calculated very idealistically which does not take in to account the practical and onsite working conditions. Considering the evolution in construction industry and analyzing the perils, it is imperative now to incorporate human reliability analysis along with structural reliability analysis as different industries are already accepting it to expeditiously manage their professional working. Among different techniques, this study recommends the model of HEART for Human Reliability Assessment. The reason for selecting this model is that it has also been tailored before by Air Traffic Management and Railways. Further, it has more flexibility and works well with any other industry.

\section{References}

[1] Atkinson, A. R. (1999). "The role of human error in construction defects." Structural Survey 17(4): 231-236.

[2] Bea, R. (2006). "Reliability and Human Factors in Geotechnical Engineering." Journal of Geotechnical and Geoenvironmental Engineering 132(5): 631-643.

[3] Bell, J. and J. Holroyd Review Of Human Reliability Assessment Methods, Health and Safety Laboratory Harpur Hill, Buxton, Derbyshire SK17 9JN 2009 
[4] Blackman, H. S., D. I. Gertman, et al. (2008). Human error quantification using performance shaping factors in the SPAR-H method, Human Factors and Ergonomics Society.

[5] Boring, R. L. and H. S. Blackman (2007). The origins of the SPAR-H method's performance shaping factor multipliers, IEEE.

[6] DiMattia, D. G., F. I. Khan, et al. (2005). "Determination of human error probabilities for offshore platform musters." Journal of Loss Prevention in the Process Industries 18(4-6): 488-501.

[7] Edmunds, J., W. Huw Gibson, et al. (2008). Nuclear Action Reliability Assessment (Nara), Further Development of a Data-Based Hra Tool. Contemporary Ergonomics 2008, Taylor \& Francis: 164-169.

[8] El-Shahhat, A. M. (1995). "Accounting for human error during design and construction." Journal of Architectural Engineering 1: 84.

[9] Epaarachchi, D. C. and M. G. Stewart (2004). "Human Error and Reliability of Multistory Reinforced-Concrete Building Construction." Journal of Performance of Constructed Facilities 18(1): 12-20.

[10] Frangopol, D. M. (1986). "Combining human errors in structural risk analysis." Civil Engineering Systems 3(2): 93 - 99.

[11] Gibson, W. and B. Kirwan (2006). "Application of the CARA HRA Tool to Air Traffic Management Safety Cases." The University of Birmingham, Birmingham, UK, Eurocontrol, Bretigny, France.

[12] Grozdanovic, M. (2005). " Usage of Human Reliability Quantification Methods." International Journal of Occupational Safety and Ergonomics 11(2): 153-159.

[13] Gue, S. S. and Y. C. Tan (2007). "Landslides: Abuses of The Prescriptive Method." International Conference on Slope 2006 Kuala Lumpur.

[14] Haydl, H. M. and A. W. Nikiel (2000). "Design and Construction Errors---Case Studies." Practice Periodical on Structural Design and Construction 5(3): 126-130.

[15] J.W. Kim, W. Jung, et al. (2006). "A Case Study for the Selection of a Railway Human Reliability Analysis Method." International Railway Safety Conference Belfast.

[16] Jamaluddin, T. A. (2006). "Human Factors and Slope Failures in Malaysia, ." Bulletin of the Geological Society of Malaysia(52): 75-84.

[17] Janney, J. R. (1986). Guide to investigation of structural failures, with editorial revisions incorporated, Amer Society of Civil Engineers.

[18] JKR (2009). "National Slope Master Plan " Sectoral Report Research and Development Jabatan Kerja Raya Malaysia.

[19] Josephson, P. E. and Y. Hammarlund (1996a). Kvalitetsfelkostnader in the 90's - A study of seven building projects. Part I: Results. Gothenburg: (Report 49th In Swedish), Chalmers University, Inst. construction economy

[20] Josephson, P. E. and Y. Hammarlund (1996b). Kvalitetsfelkostnader in the 90's - A study of sven building projects. Part II: Methodology. Gothenburg: (Report 50th In Swesub), Chalmers University, Inst. for the construction economy and construction organization.

[21] Kirwan, B. (1994). A Guide To Practical Human Reliability Assessment Taylor and Francis Group,

[22] Kirwan, B. (1996). "The validation of three human reliability quantification techniques -- THERP, HEART and JHEDI: Part 1 -technique descriptions and validation issues." Applied Ergonomics 27(6): 359-373.

[23] Kirwan, B. (1998). "Human Error Identification Techniques for Risk Assessment for High Risk System Part 1: Review and Evaluation of Techniques,." Applied Ergonomics 29(3): 157-177.

[24] Kirwan, B., R. Kennedy, et al. (1997). "The validation of three Human Reliability Quantification techniques -- THERP, HEART and JHEDI: Part II -- Results of validation exercise." Applied Ergonomics 28(1): 17-25.

[25] Kirwan, B., S. Scannali, et al. (1996). "A case study of a human reliability assessment for an existing nuclear power plant." Applied Ergonomics 27(5): 289-302.

[26] Lind, N. C. (1982). "Models of human error in structural reliability." Structural Safety 1(3): 167-175.

[27] Long, L., X. Hongwei, et al. (2009). Qualitative Simulation and Its Application Framework in Human Reliability Analysis. Intelligent Systems and Applications, 2009. ISA 2009. International Workshop on.

[28] Matousek, M. (1977). Outcomings of a survey on 800 construction failures.

[29] Melchers, R. E. (1984). "Human error in structural reliability assessments." Reliability Engineering 7(2): 61-75.

[30] Morgenstern, N. R. (1995). Managing risk in geotechnical engineering. 10t $\mathrm{t}_{\mathrm{h}}$ Pan American Conference on Soil Mechanics and Foundation Engineering.

[31] Mosleh, A. and Y. H. Chang (2004). "Model-based human reliability analysis: prospects and requirements." Reliability Engineering \& System Safety 83(2): 241-253.

[32] Mosleh, A. and K. Groth (2009). A Data-Informed Model Of Performance Shaping Factors And Their Interdependencies For use In Human Reliability Analysis. Reliability, Risk, and Safety, CRC Press.

[33] Peck, R. B. (1969). "Advantages and Limitations of the Observational Method in Soil Mechanics." Geeotechnique 19: $171-187$.

[34] Rasmussen, J. (1982). "Human errors. A taxonomy for describing human malfunction in industrial installations." Journal of Occupational Accidents 4(2-4): 311-333.

[35] Reason, J. (1990). Human error : , Cambridge University Press Cambridge.

[36] Redmill, F., T. Anderson, et al. (2007). CARA: A Human Reliability Assessment Tool for Air Traffic Safety Management Technical Basis and Preliminary Architecture. The Safety of Systems, Springer London: 197-214.

[37] Structural Engineering Documents 5., International Association for Bridges and Structural Engineering (IABSE).

[38] Smith, D. W. (1977). "Why Do Bridges Fail—." Civil Engineering-ASCE 47(11): 58-62.

[39] Sowers, G. F. (1993). "Human Factors in Civil and Geotechnical Engineering Failures." Journal of Geotechnical Engineering 119(2): 238-256.

[40] Stewart, M. G. and R. E. Melchers (1989). "Checking Models in Structural Design " J. Struct. Engrg 115(6): 1309-1324.

[41] Swain, A. D. and H. E. Guttmann (1983). Handbook on Human Reliability Analysis with Emphasis on Nuclear Power Plant Application. NUREG/CR-1278. SAND 80-0200 RX, AN. .

[42] Thornton, C. H. (1985). Failure statistics categorized by cause and generic class, ASCE New York.

[43] Williams, J. C. (1986). "HEART - A proposed method for assessing and reducing human error." Proceedings of the 9th Advances in Reliability Technology Symposium,University of Bradford.

[44] Williams, J. C. (1988). A data-based method for assessing and reducing human error to improve operational performance. Fourth Conference on Human Factors and Power Plants, Location: Monterey, CA, USA, IEEE, New York.

[45] Yates, J. K. and E. E. Lockley (2002). "Documenting and analyzing construction failures." Journal of Construction Engineering and Management 128: 8 . 